\title{
Model Associated with the Study of the Degradation Based on the Accelerated Test: A Literature Review
}

\author{
Fatou Dia, Nacire Mbengue, Omar Ngalla Sarr, Moulaye Diagne, Omar A. Niasse, \\ Awa Dieye, Mor Niang, Bassirou Ba, Cheikh Sene \\ Laboratory of Semiconductor and Solar Energy, Department of Physics, Faculty of Science and Techniques, \\ University Cheikh Anta Diop, Dakar, Senegal \\ Email: fatou9.dia@ucad.edu.sn
}

Received 19 December 2015; accepted 24 January 2016; published 27 January 2016

Copyright (C) 2016 by authors and Scientific Research Publishing Inc.

This work is licensed under the Creative Commons Attribution International License (CC BY). http://creativecommons.org/licenses/by/4.0/

\section{Open Access}

\section{Abstract}

Most manufacturers of solar modules guarantee the minimum performance of their modules for 20 to 25 years, and 30-year warranties have been introduced. The warranty typically guarantees that the modules will perform to at least $90 \%$ capacity in the first 10 years and to at least $80 \%$ in the following 10 - 15 years. Early degradation resulting from design flaws, materials or processing issues is often apparent from startup to the first few years in service. Importantly, many module failures and performance losses are the result of gradual accumulated damage resulting from long-term outdoor exposure in harsh environments, referred. Many of these processes occur on relatively long time scales and the various degradation processes may be chemical, electrical, thermal or mechanical in nature. These are either initiated or accelerated by the combined stresses of the service environment, in particular solar radiation, temperature and moisture, and other stresses such as salt air, wind and snow. Accelerated Life Testing (ALT) test methodology is normally predicated on first being able to reproduce a specific degradation or failure mode without altering it (correlation); and, second, to produce that result in less than real-time acceleration. Degradation and failure may result when an applied stress exceeds material or product strength. This may be a one-time catastrophic event, the result of cyclic fatigue, or a gradual decline in requisite properties due to ageing mechanisms. Engineers in the manufacturing industries have used accelerated test (AT) experiments for many decades. The purpose of AT experiments is to acquire reliability information quickly. Test units of a material, component, subsystem or entire systems are subjected to higher-than-usual levels of one or more accelerating variables such as temperature or stress. Then the AT results are used to predict life of the units at use conditions. The extrapolation is typically justified (correctly or incorrectly) on the basis of physically motivated models or a combination of empirical model fitting with a sufficient amount of previous experience in testing similar units. The need to extrapolate in both time and the accelerating variables 
generally necessitates the use of fully parametric models. Statisticians have made important contributions in the development of appropriate stochastic models for AT data [typically a distribution for the response and regression relationships between the parameters of this distribution and the accelerating variable(s)], statistical methods for AT planning (choice of accelerating variable levels and allocation of available test units to those levels) and methods of estimation of suitable reliability metrics. This paper provides a review of many of the AT models that have been used successfully in this area.

\title{
Keywords
}

\author{
Degradation, Acceleration Factor, Arrhenius Relationship, Eyring Relationship, Inverse Power \\ Relationship, Voltage-Stress, Photodegradation
}

\section{Introduction}

Today's manufacturers face strong pressure to develop new, higher-technology products in record time, while improving productivity, product field reliability and overall quality. This has motivated the development of methods like concurrent engineering and encouraged wider use of designed experiments for product and process improvement. The lack of accurate information about degradation rate increases the financial risk [1]. The reliability and lifetime of a photovoltaic system depend mainly on the energy performance of the modules and their different degradation mode [2]. A common misconception is since PV has a track record dating from the 1970's, that module reliability is now a given [3]. According to literature, results from experimental tests are given. Different techniques and testing methods are used. Most of these results obtained from laboratory tests are not always accurate and consistent on the degradation rates of PV modules announced. In 2002, the National Renewable Energy Laboratory (NREL) estimated the annual performance degradation of monocrystalline and polycrystalline PV modules at $0.7 \%$ [4]. John $\mathrm{H}$. Wohlgemuth, formerly with BP Solar and now with NREL, describes the fundamental problem: Outdoor testing is a must, but it takes much too long to be of much use as decision maker. We clearly cannot wait 25 years or even a significant fraction of 25 years to introduce a new product. Therefore, we must develop and utilize accelerated tests to qualify these new products. Akira Terao, Chief Reliability Engineer for Sun power Corporation goes further to identify some of the life-testing roadblocks: 25 year warranty, Ill-defined field conditions, Harsh and varied outdoor conditions, Materials used near their limits; Limited acceleration factors $\Rightarrow$ long tests, Large samples, small sample sizes; Subtle polymer chemistry; Cumulative effects, positive feedback loops. Clearly, assessing PV module lifetime performance is not a simple task. The LEEETISO (Laboratory of Energy, Ecology and Economy Solar-Ticino), test center of photovoltaic modules in Switzerland, stated that the power degradation rate of crystalline silicon PV modules could go from $0.7 \%$ to $9.8 \%$ during the first exposure year and $0.7 \%$ to $4.9 \%$ during the second one [5]. Also, that early problems, such as encapsulate yellowing or TCO corrosion are now all resolved. However, since circa 2005, silicon wafer thickness has been greatly reduced, new lower cost materials have emerged, and other changes have been introduced making today's c-Si modules very different from previous designs. And non-crystalline silicon PV technologies have different failure modes where prior silicon history isn't useful. Jordan [6] states that in the 2000s, degradation rate measured on individual modules was $5 \%$ on average. There are only a few long-term studies on the degradation of PV modules published. Skoczek [7] have measured the performance of 204 field-aged crystalline Si based PV modules (53 module types). Exposing started in 1983 at the Joint Research Center in north Italy with a moderate subtropical climate $\left(-10\right.$ to $\left.35^{\circ} \mathrm{C},>90 \% \mathrm{RH}\right)$. Today, this performance warranty is frequently $>90 \%$ of rated power after 10 years and $>80 \%$ after 20 or 25 years although 30 year guarantees have now appeared. These high performance losses $(>20 \%)$ are related to losses of the fill factor, caused by an increased series resistance. The moderate performance losses $(<20 \%)$ can be related to losses of the short-circuit current, caused by degradation of the optical properties. The long term losses are determined to be between $0.2 \%$ and $1.0 \%$ per annum.

However, there is little information on PV modules degradation modes in terms of frequency, speed of evolution and degree of impact on module lifetime and reliability. 
Research on photovoltaic modules is rather focused on the race to develop new technologies without sufficient experience feedback on already operational technologies [8] [9]. The requirements for higher reliability have increased the need for more up-front testing of materials, components and systems. This is in line with the modern quality philosophy for producing high-reliability products: achieve high reliability by improving the design and manufacturing processes; move away from reliance on inspection (or screening) to achieve high reliability, as described in Meeker and Hamada [10] and Meeker and Escobar [11] estimating the failure-time distribution or long-term performance of components of high-reliability products is particularly difficult. Most modern products are designed to operate without failure for years, decades or longer. Thus few units will fail or degrade appreciably in a test of practical length at normal use conditions.

For such applications, Accelerated Tests (ATs) are used in manufacturing industries to assess or demonstrate component and subsystem reliability, to certify components, to detect failure modes so that they can be corrected, to compare different manufacturers, and so forth. ATs have become increasingly important because of rapidly changing technologies, more complicated products with more components, higher customer expectations for better reliability and the need for rapid product development. There are difficult practical and statistical issues involved in accelerating the life of a complicated product that can fail in different ways. Generally, information from tests at high levels of one or more accelerating variables (e.g., use rate, temperature, voltage or pressure) is extrapolated, through a physically reasonable statistical model, to obtain estimates of life or longterm performance at lower, normal levels of the accelerating variable(s).

The present article proposes a bibliographic review on the degradation modes of PV modules [12]-[14] as well as the associated models for the study of the degradation of their power based on di-paid whose accelerated approaches used to determine the parameters.

The paper is organized in two parts, the main types of degradation of the PV module identified in the literature are exposed in the first part. A review of models associated with the degradation of the PV modules is presented in the second part.

\section{Modes and Causes of Degradation of a PV Module}

Manufacturer's guarantees of up to 20 years indicate the quality of bulk silicon PV modules currently being produced. Nevertheless, there are several failure modes and degradation mechanisms which may reduce the power output or cause the module to fail. Nearly all of these mechanisms are related to water ingress or temperature stress. Degradation mechanisms may involve either a gradual reduction in the output power of a PV module over time or an overall reduction in power due to failure of an individual solar cell in the module. They can deteriorate or become defective even when they operate on actual site for extended periods [15].

The degradation of PV modules are caused by static and dynamic mechanical loads, thermal cycling, exposure to radiation, moisture, hail impact (sand) dust accumulation, partial occultation causes found above are responsible for the degradation of the modules, which manifests itself in several ways:

Corrosion of the PV module, the discoloration, delamination, breakage of the front panel (glass) module, Potential Induced Degradation (PID), hot spots (hot spots), Bubbles, dust deposits.

\section{Method for Determination of the Degradation}

The outdoor exhibition of photovoltaic modules includes several factors (wind, rain, light, heat ....) That causes damage as mentioned above. Thus these factors have a negative effect on the module power output.

To better understand these degradations, called accelerated tests can reproduce they are conducted in climatic chambers in extensive conditions of temperature and pressure to reproduce the degradation in a limited time. But it is also interesting to have a model to simulate numerically the evolution of degradation. Many authors have tried to make the degradation assessment models

\subsection{The Model of Pan}

Pan [16] offers the general degradation model of a photovoltaic module following

$$
D(t)=1-\exp \left(-b t^{a}\right)
$$

where $a$ and $b$ are parameters that can be assessed by degradation testing. The parameter a is considered constant 
regardless of the level of sévérisation and b is variable reflecting its dependence on sévérisation levels. Thanks to this relationship, we can determine when the test must be stopped in order to measure the power of the module. Wohlgemuth [17] achieved a damp heat test at a temperature of $85^{\circ} \mathrm{C}$ and a relative humidity of $85 \%$, which corresponds to the sévérisée condition that we advocate and which is that of 61215 IEC standards IEC 61646 and IEC 62108. The author followed the power loss of the photovoltaic module during the test and obtained the curve shown in Figure 1. The values of $a$ and $b$ are estimated respectively: $a=3.0868$ and $b=5.762 \times 10^{-12}$.

Temperatures taken during the tests shall not exceed the technological limit temperature of PV module sequal to $120^{\circ} \mathrm{C}$ according to Kern [18].

The $85 \mathrm{C} / 85 \%$ relative humidity exposure is as accelerated as necessary. These conditions probably never happen in the real world as the modules tend to dry out at their highest temperatures, but absorb moisture at lower temperatures. It is difficult to judge what outdoor exposure the 1000 hour exposure at $85 / 85$ represents. In a recent experiment 10 crystalline silicon modules qualified to IEC 61215 were exposed to 1250 hours of 85/85. Only 2 of the 10 types successfully passed the extended test [18]. On the other hand some glass-glass encapsulated modules can easily endure more than 2000 hours of damp heat exposure. So rather than specify a particular length of time, it seems appropriate to test the control technology and the new technology through enough hours of damp heat that both begin to lose some power (say to $90 \%$ of the original) in order to verify that the new technology is no worse and has no additional failure modes than the old module technology it will replace.

The study of Wohlgemuth [17] to determine the parameters of the degradation model proposed by Pan [16]: $a$ $=3.0868$ and $b=5.762 \times 10^{-12}$ for a temperature of $85^{\circ} \mathrm{C}$ and a relative humidity $85 \%$. The parameter is considered constant whatever the test conditions (temperature and humidity) while the parameter $b$ depends on the test conditions. For the model of Peck, $b$ of the degradation model parameter to a temperature $T$ and relative humidity RH can be determined by the relation

$$
b=b_{85^{\circ} \mathrm{C} / 85 \%} \cdot \mathrm{e}^{a \cdot n \cdot \ln \left(\frac{H R}{0.85}\right)-a \frac{E_{a}}{k} \cdot\left(\frac{1}{T}-\frac{1}{358}\right)}
$$

or $b_{85^{\circ} \mathrm{C} / 85 \%}$ is the parameter $b$ of degradation model for a temperature of $358^{\circ} \mathrm{K}\left(85^{\circ} \mathrm{C}\right)$ and a relative humidity of $85 \%$. The parameter b of the acceleration model is equal to $1.948 \times 10^{-9}$ for a temperature of $105^{\circ} \mathrm{C}$ and a relative humidity of $85 \%$, and equalto $1.614 \times 10^{-11}$ for conditions of $85^{\circ} \mathrm{C} / 95 \% \mathrm{HR}$.

The constants $a$ and $b$ depend on the degradation mode considered. Thus, Equation (2) and (3) give respectively the degradation model due to corrosion and discoloration:

$$
\begin{aligned}
& D_{\text {corrosion }}(t)=1-\exp \left(-b_{\text {corrosion }} \cdot t^{a_{\text {corrosion }}}\right) \\
& D_{\text {decoloration }}(t)=1-\exp \left(-b_{\text {decoloration }} \cdot t^{a_{\text {decoloration }}}\right)
\end{aligned}
$$

where $a$ ( $a$ corrosion and $a$ discoloration) and $b$ ( $b$ corrosion and $b$ discoloration) are the parameters of the degradation model. They are determined from accelerated testing Charki, and Laronde [19]-[21].

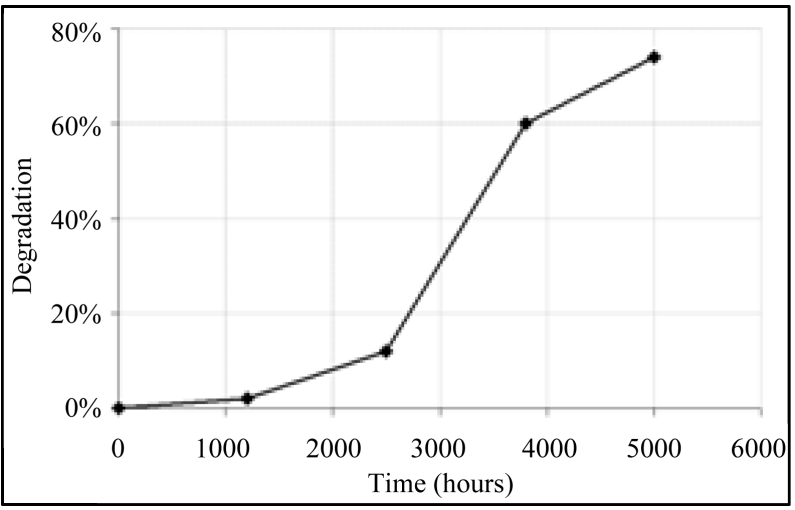

Figure 1. Dégradation d'un module en polycristallin-Si en exposition $85 / 85$. 


$$
D_{\text {degradation module }}(t)=1-\prod_{k=1}^{n}\left(1-D_{k}(t)\right)
$$

where $n$ is the number of degradation modes.

\subsection{The Exponential Model}

The PV module power measured at a given time follows a Gaussian distribution. According to Reis, Sakamoto and Oshiro [18] [22], the probability density is given by:

$$
P(p)=\left(\frac{1}{\sqrt{2 \pi \sigma}} \exp \left(-\frac{1}{2}\right)\left[\left(\frac{P-\mu}{\sigma}\right)\right]^{2}\right)
$$

where $P$ is the power module, $\mu$ is its mean value and $\sigma$ its standard deviation. Thus, it can be calculated by Osterwald, Marion, Adelstein, and Raghuraman [23]-[25]:

$$
\mu(t)=P_{0}-A t
$$

where

$P_{0}$ is the average power at $t=0$ (i.e. the nominal module power),

$A$ is a parameter which reflects annual decrease of module power,

$t$ is time in years. Of course, validity of Equation (6) is restricted by time $(t)$ lower than $P_{0} / A$.

Another limitation comes from the assumption according to which $A$ is constant in time.

$$
\frac{\mu(n)-\mu(n-1)}{P_{0}}=-\frac{A}{P_{0}}
$$

$A / P_{0}\left(\right.$ year $^{-1}$ ) is the annual degradation rate. From studies on electronic components degradation, we can consider the assumption according to which degradation rate is exponential [26] [27]

$$
\mu(t)=P_{0} \exp (-a t)
$$

Figure 2 shows PV module degradation as a function of time from the exponential model.

\subsection{Using Temperature to Accelerate Failure Mechanisms}

It is sometimes said that high temperature is the enemy of reliability. Increasing temperature is one of the most commonly used methods to accelerate a failure mechanism.

\subsubsection{Arrhenius Relationship for Reaction Rates}

The Arrhenius relationship is a widely used model to describe the effect that temperature has on the rate of a simple chemical reaction. This relationship can be written as

$$
R(\text { temp })=\gamma_{0} \exp \left(\frac{-E a}{k t e m p K}\right)
$$

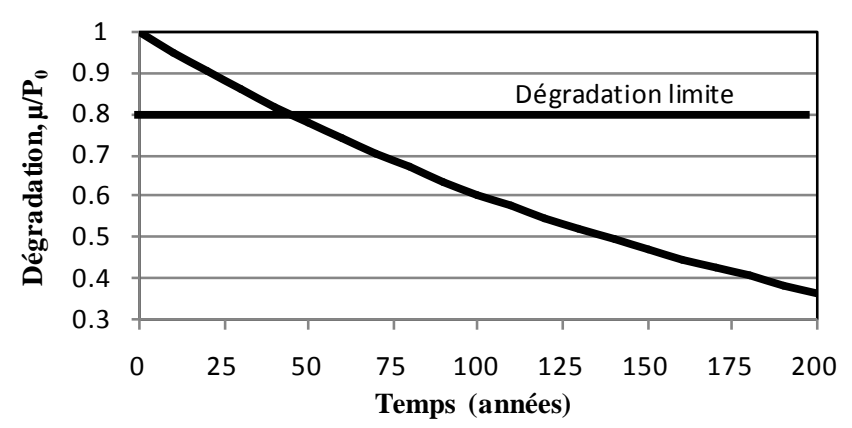

Figure 2. Exponential degradation of PV module power [28]. 
where $R$ is the reaction rate, and temp ${ }^{\circ} \mathrm{K}=$ temp ${ }^{\circ} \mathrm{C}+273.15$ is thermodynamic temperature in kelvin $(\mathrm{K}), k$ is either Boltzmann's constant or the universal gas constant and $E a$ is the activation energy.

The parameters $E a$ and $\gamma_{0}$ are product or material characteristics. In applications involving electronic component reliability, Boltzmann's constant $k=8.6171 \times 10^{-5}=1 / 11605$ in units of electron-volt per kelvin $(\mathrm{eV} / \mathrm{K})$ is commonly used and in this case, $E a$ has units of electron-volt $(\mathrm{eV})$.

In most accelerated test applications, it would be more appropriate to refer to Ea in Equation (9) as a quasiactivation energy.

\subsubsection{Arrhenius Relationship Time-Acceleration Factor}

The Arrhenius acceleration factor is

$$
A F\left(\text { temp }, \text { temp }_{U}, E a\right)=\frac{R(\text { temp })}{R\left(\text { temp }_{U}\right)}=\exp \left[E a\left(\frac{11605}{\text { temp }_{U} K}-\frac{11605}{\text { tempK }}\right)\right]
$$

When temp $>$ temp $_{U}, A F\left(\right.$ temp, temp $\left.p_{U}, E a\right)>1$.

When temp $p_{U}$ and Ea are understood to be, respectively, product use temperature and reaction-specific quasi activation energy, $A F($ temp $)=A F\left(\right.$ temp , temp $\left.p_{U}, E a\right)$ will be used to denote a time-acceleration factor.

The Arrhenius-based model presents some limits. Indeed, the Arrhenius equation can be used to quantify the effect of varying temperature and irradiance on the rate of a property change. However it cannot provide a complete picture of the long-term degradation of PV modules, as other stress factors or combination of stresses are involved. These include moisture (inducing physical and chemical processes and generating mechanical stress in combination with temperature), temperature cycling (generating thermomechanical stress), electricity production (inducing electrical and electrochemical stresses), and other externally applied stresses (wind, hail, airborne pollutants.

\subsubsection{Eyring Relationship Time-Acceleration Factor}

The Arrhenius relationship Equation (9) was discovered by Svante Arrhenius through empirical observation in the late 1800s. Eyring [29] [30] gives physical theory describing the effect that temperature has on a reaction rate. Written in terms of a reaction rate,

The Eyring relationship is:

$$
R(\text { temp })=\gamma_{0} \times A(\text { temp }) \times \exp \left(\frac{-E a}{k \times t e m p K}\right)
$$

where $A(t e m p)$ is a function of temperature depending on the specifics of the reaction dynamics and 0 and Ea are constants Weston and Schwarz, provides more detail [31]. Applications in the literature have typically used $A($ temp $)=(\text { tempK })^{m}$ with a fixed value of $m$ ranging between $m=0$ [32], $m=0.5$ [33], to $m=1$ [34] [35]. The Eyring relationship temperature acceleration factor is

$$
A F_{E_{Y}}\left(\text { temp }, \text { temp }_{U}, E a\right)=\left(\frac{\text { tempK }}{\text { temp }_{U} K}\right)^{m} \times A F_{A r}\left(\text { temp }, \text { temp }_{U}, E a\right)
$$

where $A F_{A r}\left(t e m p, t e m p_{U}, E a\right)$ is the Arrhenius acceleration factor from Equation (10) For use over practical ranges of temperature acceleration, and for practical values of $m$ not far from 0 , the factor outside the exponential has relatively little effect on the acceleration factor and the additional term is often dropped in favor of the simpler Arrhenius relationship.

\subsection{Using Humidity to Accelerate Reaction Rates}

Humidity is another commonly used accelerating variable, particularly for failure mechanisms involving corrosion and certain kinds of chemical degradation.

Vapor density measures the amount of water vapor in a volume of air in units of mass per unit volume.

Partial vapor pressure (sometimes simply referred to as "vapor pressure") is closely related and measures that part of the total air pressure exerted by the water molecules in the air. Partial vapor pressure is approximately 
proportional to vapor density.

The partial vapor pressure at which molecules are evaporating and condensing from the surface of water at the same rate is the saturation vapor pressure.

For a fixed amount of moisture in the air, saturation vapor pressure increases with temperature.

Relative humidity is usually defined as and is commonly expressed as a percent. For most failure mechanisms, physical/chemical theory suggests that RH is the appropriate scale in which to relate reaction rate to humidity especially if temperature is also to be used as an accelerating variable [33].

A variety of different humidity models (mostly empirical but a few with some physical basis) have been suggested for different kinds of failure mechanisms.

$$
\mathrm{RH}=\frac{\text { Vapor Pressure }}{\text { Saturation Vapor Pressure }}
$$

Much of this work has been motivated by concerns about the effect of environmental humidity on plasticpackaged electronic devices. Humidity is also an important factor in the service-life distribution of paints and coatings. In most test applications where humidity is used as an accelerating variable, it is used in conjunction with temperature.

Peck [36] presents data and models relating life of semiconductor electronic components to humidity and temperature. See also Peck and Zierdt [37] and Joyce et al. [38]. Gillen and Mead [39] describe a kinetic approach for modeling accelerated aging data. LuValle, Welsher and Mitchell [40] describe the analysis of timeto-failure data on printed circuit boards that have been tested at higher than usual temperature, humidity and voltage.

They suggest ALT models based on the physics of failure. Nelson [34] and Boccaletti et al. [32] review and compare a number of different humidity models.

The Eyring/Arrhenius temperature-humidity acceleration relationship in the form of Equation (33) uses $x_{1}=$ 11605/temp $K, x_{2}=\log (\mathrm{RH})$ and $x_{3}=x_{1} x_{2}$ where $\mathrm{RH}$ is relative humidity, expressed as a proportion.

An alternative humidity relationship suggested by Klinger [33], on the basis of a simple kinetic model for corrosion, uses the term $x_{2}=\log [\mathrm{RH} /(1-\mathrm{RH})]$ (a logistic transformation) instead.

In most applications where it is used as an accelerating variable, higher humidity increases degradation rates and leads to earlier failures. In applications where drying is the failure mechanism, however, an artificial environment with lower humidity can be used to accelerate a test.

\subsection{Acceleration Model for Photodegradation}

Many organic compounds degrade chemically when exposed to ultraviolet (UV) radiation. Such degradation is known as photodegradation. This section describes models that have been used to study photodegradation and that are useful when analyzing data from accelerated photodegradation tests. Many of the ideas in this section originated from early research into the effects of light on photographic emulsions [41] and the effect that UV exposure has on causing skin cancer [42].

Important applications include prediction of service life of products exposed to UV radiation (outdoor weathering) and fiber-optic systems.

It is clear that UV radiation is a major degradation factor for photovoltaic modules especially in their discoloration [43]-[45]. The total dose of UV radiation can be considered as the number of photons absorbed by the material degrading and that will cause its chemical change. For PV module, this degradation is reflected by the change of the encapsulating module transmittance that induces a decrease in the PV module current-voltage characteristic. This decrease is quantified by the relative variation of module short-circuit current representing the degradation factor given by Zimmerman [46]:

$$
D(E)=\frac{I_{s c}(E)}{I_{s c}(E=0)}
$$

where $I_{s c}(E)$ is the short-circuit current of the PV module and $E$ the dose of ultra-violet (UV). Zimmerman [46] proposes the degradation expression factor given by the following equation: 


$$
D(E)=\frac{\int_{\lambda \min }^{\lambda \max } S R(\lambda) T_{c m x}(\lambda) P(\lambda) T(\lambda, E) \mathrm{d} \lambda}{\int_{\lambda \min }^{\lambda \max } S R(\lambda) T_{c m x}(\lambda) P(\lambda) T_{0}(\lambda) \mathrm{d} \lambda}
$$

where $S R(\lambda)$ is the spectral response of PV cell, $T(\lambda, E)$ and $T_{c m x}(\lambda)$ respectively represent the transmittances of the encapsulant and the glass slide of PV module, $T_{0}(\lambda)$ is the transmittance of not irradiated PV cell. $P(\lambda)$ is the spectral power density of the sun. $\left[\lambda_{\min }, \lambda_{\max }\right]$ represents the integration interval for the wavelength range in which the spectral response of the PV cell is not zero. For a Photodegradation $T(\lambda, E) / T_{0}(\lambda)$ in the encapsulant less than $70 \%$ in the range $\left[k_{\min }, k_{\max }\right]$, the transmittance can be written:

$$
T(\lambda, E)=T_{0}(\lambda)\left[1-b_{c m x}(\lambda) \ln \left(1+a_{c m x} E\right)\right]
$$

where $a_{c m x}$ and $b_{c m x}$ are parameters of material used for PV cell [46].

The relationship between $\mathrm{UV}$ dose $E$ and exposure time $\mathrm{t}$ in the solar spectrum $P(k)$ is:

$$
E=c t \quad \text { avec } \quad c=\int_{0}^{400} T_{c m x}(\lambda) P(\lambda) \mathrm{d} \lambda
$$

The integral extends up to $400 \mathrm{~nm}$ that represents a practical limit to UV photodegradation.

Combining Equation (15) and (17) and using the mean value theorem, Equation (18) obtained to estimate the UV degradation of PV module is given by:

$$
D(t)=1-n \ln \left(1+a_{c m x} c t\right)
$$

Avec $n=b_{c m x}(\lambda), \lambda \in\left[\lambda_{\min }, \lambda_{\max }\right]$ et $t$ le temps, where $n=b_{c m x}(\lambda), \lambda \in\left[\lambda_{\min }, \lambda_{\max }\right]$ and $\left[\lambda_{\min }, \lambda_{\max }\right]$ represents the integration interval for the wavelength range where the spectral response of PV cell is not zero.

This model presents a major constraint. In fact, its use requires knowledge of the intrinsic characteristics of the materials used for the production of PV cells such as $a_{c m x}, b_{c m x}, S R(\lambda), T(\lambda, E), T_{c m x}(\lambda)$ and $T_{0}(\lambda)$. The measurement of these characteristics requires a rigorous instrumentation without which the model accuracy is compromised.

\subsubsection{Time Scale and Model for Total Effective UV Dosage}

As described in Martin et al. [47], the appropriate time scale for photodegradation is the total (i.e., cumulative) effective UV dosage, denoted by $D_{\text {Tot }}$. Intuitively, this total effective dosage can be thought of as the cumulative number of photons absorbed into the degrading material and that cause chemical change. The total effective UV dosage at real time $t$ can be expressed as

$$
D_{\text {Tot }}(t)=\int_{0}^{t} D_{\text {Inst }}(\tau) \mathrm{d} \tau
$$

where the instantaneous effective UV dosage at real time $\tau$ is

$$
D_{\text {Inst }}(\tau)=\int_{\lambda_{1}}^{\lambda_{2}} D_{\text {Inst }}(\tau, \lambda) \mathrm{d} \lambda=\int_{\lambda_{1}}^{\lambda_{2}} E_{0}(\tau, \lambda) \times\{1-\exp [-A(\lambda)]\} \phi(\lambda) \mathrm{d} \lambda
$$

Here $E_{0}(\tau, \lambda)$ is the spectral irradiance (or intensity) of the light source at time $\tau$ (both artificial and natural light sources have potentially time-dependent mixtures of light at different wavelengths, denoted by $\lambda$ ), $[1-\exp (-A(\lambda))]$ is the spectral absorbance of the material being exposed (damage is caused only by photons that are absorbed into the material), and $\phi(\lambda)$ is a quasi-quantum efficiency of the absorbed radiation (allowing for the fact that photons at certain wavelengths have a higher probability of causing damage than others). The functions $E_{0}$ and $A$ in the integrand of Equation (20) can either be measured directly or estimated from data and the function $\phi(\lambda)$ can be estimated from data. A simple log-linear model is commonly used to describe quasi-quantum efficiency as a function of wavelength. That is:

$$
\phi(\lambda)=\exp \left(\beta_{0}+\beta_{1} \lambda\right)
$$


The integrals over wavelength, like that in Equation (20), are typically taken over the UV-B band (290 nm to $14,320 \mathrm{~nm})$, as this is the range of wavelengths over which both $\phi(\lambda)$ and $E_{0}(t, \lambda)$ are importantly different from 0 . Longer wavelengths (in the UV-A band) are not terribly harmful to organic materials $[\phi(\lambda) \approx 0]$. Shorter wavelengths (in the UV-C band) have more energy, but are generally filtered out by ozone in the atmosphere $\left[E_{0}(t, \lambda) \approx 0\right]$.

\subsubsection{Model for Photodegradation and UV Intensity}

Degradation (or damage) $D(t)$ at time $t$ depends on environmental variables like $\mathrm{UV}$, temp and $\mathrm{RH}$, that may vary over time, say according to a multivariable profile $\xi(t)=[\mathrm{UV}$, temp, RH, $\cdots]$. Laboratory tests are conducted in well-controlled environments, usually holding these variables constant (although sometimes such variables are purposely changed during an experiment, as in step-stress accelerated tests). Interest often centers, however, on life in a variable environment. These sample paths might be modeled by a given functional form,

$$
D(t)=g(z), \quad z=\log [d(t)]-\mu
$$

where $z$ is scaled time and $g(z)$ would usually be suggested by knowledge of the kinetic model (e.g., linear for zeroth-order kinetics and exponential for first-order kinetics), although empirical curve fitting may be adequate for purposes where the amount of extrapolation in the time dimension is not large. As in SAFT models, $\mu$ can be modeled as a function of explanatory variables like temperature and humidity when these variables affect the degradation rate.

\subsection{Inverse Power Relationship}

The inverse power relationship is frequently used to describe the effect that stresses like voltage and pressure have on lifetime. Voltage is used in the following discussion. When the thickness of a dielectric material or insulation is constant, the voltage stress is proportional to the square of the voltage. Let volt denote voltage and let volt $_{U}$ be the voltage at use conditions. The lifetime at stress level volt is given by

$$
T(\text { volt })=\frac{T\left(\text { volt }_{U}\right)}{A F(\text { volt })}=\left(\frac{\text { volt }}{\text { volt }_{U}}\right)^{\beta_{1}} T\left(\text { volt }_{U}\right)
$$

where $\beta_{1}$, in general, is negative. The 1 model has SAFT form with acceleration factor

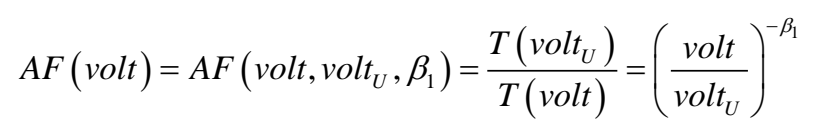

If $T\left(\right.$ volt $\left._{U}\right)$ has a log-location-scale distribution with parameters $\mu_{U}$ and $\sigma$, then $T($ volt $)$ also has a log locationscale distribution with $\mu=\beta_{1}+\beta_{1 x}$, where $x_{U}=\log \left(\right.$ volt $\left._{U}\right), x=\log ($ volt $), \beta_{0}=\mu_{U}-\beta_{1 x_{U}}$ and $\sigma$ does not depend on $x$.

The inverse power relationship is widely used to model life as a function of pressure-like accelerating variables (e.g., stress, pressure, voltage stress).

This relationship is generally considered to be an empirical model because it has no formal basis from knowledge of the physics/chemistry of the modeled failure modes. It is commonly used because engineers have found, over time, that it often provides a useful description of certain kinds of AT data.

Analytically, suppose that degrading dielectric strength at age t can be expressed as

$$
\begin{gathered}
D(t)=\delta_{0} \times t^{1 / \beta_{1}} \\
T(\text { volt })=\left(\frac{\text { volt }}{\delta_{0}}\right)^{\beta_{1}}
\end{gathered}
$$

Then the acceleration factor for volt versus volt $t_{U}$ is

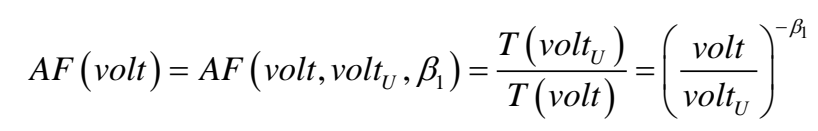


which is an inverse power relationship, as in Equation (24). To extend this model, suppose that higher voltage also leads to an increase in the degradation rate and that this increase is described with the degradation model:

$$
D(t)=\delta_{0}[R(\text { volt }) \times t]^{1 / \gamma_{1}}
$$

where $R($ volt $)=\gamma_{0} \exp \left[\gamma_{2} \log (\right.$ volt $\left.)\right]$.

Then equating $D(t)$ to volt and solving for failure time $t$ gives the failure time

$$
T(\text { volt })=\frac{1}{R(\text { volt })}\left(\frac{\text { volt }}{\delta_{0}}\right)^{\gamma_{1}}
$$

Then the ratio of failure times at volt $_{U}$ versus volt is the acceleration factor

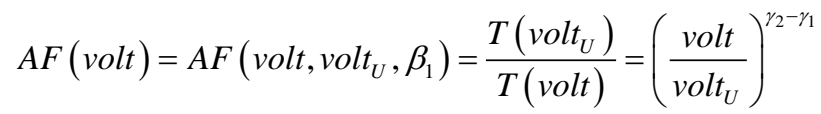

which is again an inverse power relationship with $\beta_{1}=1-2$. This motivation for the inverse power relationship described here is not based on any fundamental understanding of what happens to the insulating material at the molecular level over time.

\subsection{Acceleration Models with More than One Accelerating Variable}

\subsubsection{Generalized Eyring Relationship}

The generalized Eyring relationship extends the Eyring relationship in Section 3.3.3, allowing for one or more nonthermal accelerating variables (such as humidity or voltage). For one additional nonthermal accelerating variable $X$, the model, in terms of reaction rate, can be written as

$$
R(\text { temp }, X)=\gamma_{0} \times(\text { tempK })^{m} \times \exp \left(\frac{-\gamma_{1}}{k \times t e m p K}\right) \times \exp \left(\gamma_{2} X+\frac{\gamma_{3} X}{k \times t e m p K}\right)
$$

where $X$ is a function of the non-thermal stress. The parameters $\gamma_{1}=E a$ (activation energy) and $0,2,3$ are characteristics of the particular physical/chemical process. Additional factors like the one on the right-hand side of Equation (31) can be added for other non-thermal accelerating variables.

In the following sections, following common practice, we set $(\text { tempK })^{m}=1$, using what is essentially the Arrhenius temperature-acceleration relationship.

These sections describe some important special-case applications of this more general model. If the underlying model relating the degradation process to failure is a SAFT model, the generalized Eyring relationship can be used to describe the relationship between times at different sets of conditions temp and $X$. In particular, the acceleration factor relative to use conditions temp $p_{U}$ and $X_{U}$ is

$$
A(\text { temp }, X)=\frac{R(\text { temp }, X)}{R\left(\text { temp }_{U}, X_{U}\right)}
$$

Suppose that $T\left(\operatorname{temp}_{U}\right)$ (time at use or some other baseline temperature) has a log location scale distribution with parameters $\mu_{U}$ and $\sigma$. Then $T(t e m p)$ has the same log-location-scale distribution with

$$
\left.\mu=\mu_{U}-\log [\text { AF (temp, } X)\right]=\beta_{0}+\beta_{1} x_{1}+\beta_{2} x_{2}+\beta_{2} x_{1} x_{2}
$$

where $\beta_{1}=E_{a}, \beta_{2}=-\gamma_{2}, \beta_{3}=-\gamma_{3}, x_{1}=\frac{11605}{\text { tempK }}, x_{2}=X$ and $\beta_{0}=\mu_{U}-\beta_{1} x_{1 U}-\beta_{2} x_{2 U}-\beta_{3} x_{1 U} x_{2 U}$.

\subsubsection{Temperature-Voltage Acceleration Models}

Many different models have been used to describe the effect of the combination of temperature and voltage on acceleration. For instance, Meeker and Escobar [48] analyzed data from a study relating voltage and temperature to the failure of glass capacitors. They modeled the location parameter of log-lifetime as a simple linear function of temp ${ }^{\circ} \mathrm{C}$ and volt. The generalized Eyring relationship can also be used with $X=\log ($ volt $)$, as done in Boyko and Gerlach [49]. Klinger [33] modeled the Boyko and Gerlach [49] data by including second-order terms for 
both accelerating variables. To derive the time-acceleration factor for the extended Arrhenius relationship with temp and volt, one can follow steps analogous to those outlined in Section 3.6. Using Equation (31) with $X=$ $\log ($ volt $)$, one obtains

$$
R(\text { temp }, \text { volt })=\gamma_{0} \times \exp \left(\frac{-E a}{k \times t e m p K}\right) \times \exp \left[\gamma_{2} \log (\text { volt })+\frac{\gamma_{3} \log (\text { volt })}{k \times t e m p K}\right]
$$

Again, failure occurs when the dielectric strength crosses the applied voltage stress, that is, $D(t)=$ volt. This occurs at time

$$
T(\text { temp }, \text { volt })=\frac{1}{R(\text { temp }, \text { volt })}\left(\frac{\text { volt }}{\delta_{0}}\right)^{\gamma_{1}}
$$

From this, one computes

$$
\begin{aligned}
A F(\text { temp }, \text { volt })= & \frac{T\left(\text { temp }_{U}, \text { volt }_{U}\right)}{T(\text { temp }, \text { volt })}=\exp \left[E a\left(x_{1 U}-x_{1}\right)\right] \times\left(\frac{\text { volt }^{\text {volt }_{U}}}{\gamma_{2}-\gamma_{1}}\right. \\
& \times\left\{\exp \left[x_{1} \log (\text { volt })-x_{1 U} \log \left(\text { volt }_{U}\right)\right]\right\}^{\gamma_{3}}
\end{aligned}
$$

where $x_{1 U}=11605 /\left(\operatorname{temp}_{U} K\right)$ and $x_{1}=11605 /($ tempK $)$. When $\gamma_{3}=0$, there is no interaction between temperature and voltage. In this case, $\mathrm{AF}$ (temp, volt) can be factored into two terms, one that involves temperature only and another term that involves voltage only. Thus, if there is no interaction, the contribution of temperature (voltage) to acceleration is the same at all levels of voltage (levels of temperature).

\subsubsection{Temperature-Humidity Acceleration Models}

Relative humidity is another environmental variable that can be combined with temperature to accelerate corrosion or other chemical reactions.

Gillen and Mead [39] and Klinger [33] studied kinetic models relating aging with humidity. LuValle, Welsher and Mitchell [40] provided physical basis for studying the effect of humidity, temperature and voltage on the failure of circuit boards. See Boccaletti et al. [32], Nelson [34], Joyce et al. [38], Peck [36] and Peck and Zierdt [37] for ALT applications involving temperature and humidity.

The extended Arrhenius relationship Equation (31) applied to ALTs with temperature and humidity uses $x_{1}=$ $11605 /$ tempK, $x_{2}=\log (\mathrm{RH})$ and $x_{3}=x_{1} x_{2}$ where $\mathrm{RH}$ is a proportion denoting relative humidity. The case when $\beta_{3}=0$ (no temperature-humidity interaction) is known as "Peck's relationship" and was used by Peck [36] to study failures of epoxy packing.

Klinger [33] suggested the term $x_{2}=\log [\mathrm{RH} /(1-\mathrm{RH})]$ instead of $\log (\mathrm{RH})$. This alternative relationship is based on a kinetic model for corrosion.

The acceleration model with the capacity to take into account temperature $(T)$ and relative humidity (RH) is the Peck model [50].

According to Charki et al. [19], the Wiener process can be estimated as follows:

$$
\left(x_{i}, z_{i j}\right)=1-\exp \left(-b^{0} \cdot\left(r\left(x_{i}, \gamma\right) t_{i j}\right)^{a^{0}}\right)
$$

In this case, $x_{1}$ and $x_{2}$ correspond respectively to relative humidity $(\mathrm{RH})$ and module temperature $(T) ; x_{1}^{0}$ and $x_{2}^{0}$ respectively correspond to known relative humidity $\left(H R^{0}\right)$ and temperature $\left(T^{0}\right)$ in the reference conditions.

The acceleration factor is expressed as [19]:

$$
r\left(x_{1}, x_{2}, \gamma\right)=\exp \left(n \ln \left(\frac{H R}{H R^{0}}\right)-\frac{E a}{k}\left(\frac{1}{T}-\frac{1}{T^{0}}\right)\right)
$$

where $\gamma=(n, E a)$.

We consider that $a^{0}=a$ for each stress level. The relation Equation (36) becomes:

$$
\left(x_{i}, z_{i j}\right)=1-\exp \left(-b\left(x_{i}, \gamma\right) t_{i j}^{a}\right)
$$


where $b\left(x_{i}, \gamma\right)=b^{0} \exp \left(a \cdot n \cdot \ln \left(\frac{H R}{H R^{0}}\right)-\frac{a E a}{k}\left(\frac{1}{T}-\frac{1}{T^{0}}\right)\right)$.

If the acceleration factor is defined by relation Equation (37), it is possible to estimate an equivalent temperature, $T_{e q}$, which represents the degradation that would have occurred if the module had been aged for the same length of time but at a constant temperature. The equivalent temperature $T_{e q}$ can be calculated using the following relation [51]:

$$
\exp \left(\frac{-E a}{k \cdot T_{e q}}\right)=\left(\frac{1}{t_{2}-t_{1}}\right) \int_{t_{1}}^{t_{2}} \exp \left(\frac{-E a}{k \cdot T_{\text {module }(t)}}\right) \mathrm{d} t
$$

where $t$ is time, $T_{\text {module }(t)}$ is the time-dependent module temperature, and $t_{1}$ and $t_{2}$ are the integration start and end times.

Using the same methodology below, the equivalent of relative humidity $H_{e q}$ can be estimated as

$$
\left(H_{e q}\right)^{n}=\left(\frac{1}{t_{2}-t_{1}}\right) \int_{t_{2}}^{t_{1}}(H(t))^{n} \mathrm{~d} t
$$

where $t$ is time, $H(t)$ is the time-dependent environmental relative humidity, and $t_{1}$ and $t_{2}$ are the integration start and end times.

The module temperature $T_{\text {module(t) }}$ and the relative humidity $H(t)$ are simulated hour by hour for a period of 50 years, for at four cities: Paris (France), Oslo (Norway), Madrid (Spain) and Tamanrasset (Algeria). The equivalent temperature $T_{e q}$ and the equivalent relative humidity $H_{e q}$ obtained versus Ea and n respectively using relations Equations (39) and (40) are plotted in Figure 3.

\subsubsection{Modeling Photodegradation Temperature and Humidity Effects}

When modeling photodegradation, it is often necessary to account for the effect of temperature and humidity. The Arrhenius rate reaction model Equation (9) can be used to scale time (or dosage) in the usual manner. Humidity is also known to affect photodegradation rate. Sometimes the rate of degradation will be directly affected by moisture content of the degrading material. In this case one can use a model such as described in Burch, Martin and Van Landingham [52] to predict moisture content as a function of relative humidity.

Combining these model terms with the log of total effective UV dosage from Equation (19) gives

$$
\begin{aligned}
& \log (d ; C F, p)=\log \left[D_{\text {Tot }}(t)\right]+p \times \log (C F) \\
& \mu=\beta_{0}+\frac{E a}{(k \times t e m p K)}+C \times \mathrm{MC}(\mathrm{RH})
\end{aligned}
$$

where temp $K$ is temperature in kelvin, $\mathrm{MC}(\mathrm{RH})$ is a model prediction of moisture content, as a function of relative humidity, $k$ is Boltzmann's constant, $E_{a}$ is a quasi-activation energy, and $\beta_{0}$ and $C$ are parameters that are characteristic of the material and the degradation process.

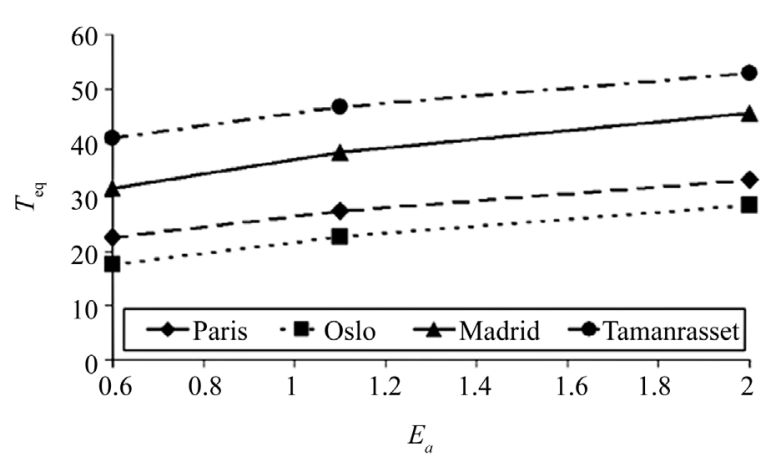

(a)

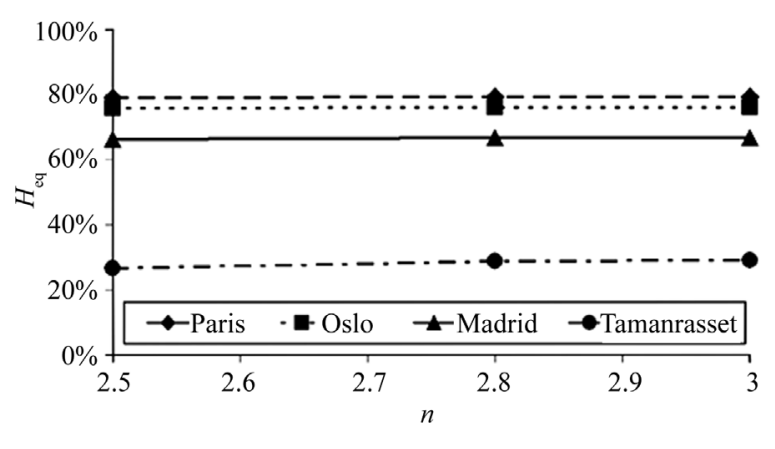

(b)

Figure 3. Equivalent temperature versus $E a$ (a) and equivalent relative humidity versus $n$ (b) for the four cities studied. 


\section{Conclusions}

Accelerated test programs often start with simple experiments to understand the failure modes that can occur and the behavior of mechanisms that can cause failures.

Use of methods for designed experiments is important for these tests. In addition, there may be special features of accelerated tests that require special test planning methods, providing expertise in the analysis of data arising from preliminary studies and the accelerated tests themselves. Features such as censoring, multiple failure modes and models that are nonlinear in the parameters are common. Methods for detecting model departures are particularly important. The work presented in this paper has underlined the degradation modes and factors to consider in experiments over long periods. Development of models associated with different degradation modes of PV modules is an interesting research field to improve the knowledge of photovoltaic modules behavior during their life cycle.

Research in the development of accelerated test models is a multidisciplinary activity.

\section{References}

[1] Jordan, D.C. (2011) Methods for Analysis of Outdoor Performance Data. NREL PV Module Reliability Workshop, Golden, CO. http://www.nrel.gov/pv/pvmrw.html

[2] Laronde, R., Charki, A. and Bigaud, D. (2012) Lifetime Estimation of a Photovoltaic Module Subjected to Corrosion Due to Damp Heat Testing. Journal of Solar Energy Engineering, 135, Article ID: 021010. http://dx.doi.org/10.1115/1.4023101

[3] Quintana, M.A., King, D.L., McMahon, T.J. and Osterwald, C.R. (2002) Commonly Observed Degradation in FieldAged Photovoltaic Modules. Conference Record of the 29th IEEE Photovoltaic Specialists Conference, New Orleans, 19-24 May 2002, 1436-1439. http://dx.doi.org/10.1109/pvsc.2002.1190879

[4] Osterwald, C.R., Anderberg, A., Rummel, S. and Ottoson, L. (2002) Degradation Analysis of Weathered CrystallineSilicon PV Modules. Conference Record of the 29th IEEE Photovoltaic Specialists Conference, New Orleans, 19-24 May 2002, 1392-1395. http://dx.doi.org/10.1109/pvsc.2002.1190869

[5] LEEE-TISO, 2008. http://leee.dct.supsi.ch/PV/Results/Tested_modules.htm

[6] Jordan, D.C. and Kurtz, S.R. (2011) Photovoltaic Degradation Rates-An Analytical Review. Progress in Photovoltaics: Research and Applications, 21, 12-29. http://dx.doi.org/10.1002/pip.1182

[7] Skoczek, A., Sample, T. and Dunlop, E.D. (2009) The Results of Performance Measurements of Field-Aged Crystalline Silicon Photovoltaic Modules. Progress in Photovoltaics: Research and Applications, 17, 227-240. http://dx.doi.org/10.1002/pip.874

[8] Laronde, R. (2009) Solutions Photovolta1ques dans le Batiment. GINGER CATED.

[9] Tiwari, G. and Dubey, S. (2010) Fundamentals of Photovoltaic Modules and Their Applications. Royal Society of Chemistry, London.

[10] Meeker, W.Q. and Hamada, M. (1995) Statistical Tools for the Rapid Development and Evaluation of High-Reliability Products. IEEE Transactions on Reliability, 44, 187-198. http://dx.doi.org/10.1109/24.387370

[11] Meeker, W.Q. and Escobar, L.A. (2004) Reliability: The Other Dimension of Quality. Quality Technology and Quantitative Management, 1, 1-25.

[12] Osterwald, C.R., Anderberg, A., Rummel, S. and Ottoson, L. (2002) Degradation Analysis of Weathered CrystallineSilicon PV Modules. Proceedings of the Twenty-Ninth IEEE Photovoltaic Specialists Conference, New Orleans, 19-24 May 2002, 1392-1395. http://dx.doi.org/10.1109/pvsc.2002.1190869

[13] http://leee.dct.supsi.ch/PV/Results/Tested_modules.htm

[14] Morita, K., Inoue, T., Kato, T., Tsuda, I. and Hishikawa, Y. (2003) Degradation Factor Analysis of Crystalline-Si PV Modules through Long-Term Field Exposure Test. Proceedings of the 3rd World Conference on Photovoltaic Energy Conversion, Osaka, 11-18 May 2003, 1948-1951.

[15] Munoz, M.A., Alonso-Garcia, M.C., Vela, N. and Chenlo, F. (2011) Early Degradation of Silicon PV Modules and Guaranty Conditions. Solar Energy, 85, 2264-2274. http://dx.doi.org/10.1016/j.solener.2011.06.011

[16] Pan, R., Kuitche, J. and Tamizhmani, G. (2011) Degradation Analysis of Solar Photovoltaic Modules: Influence of Environmental Factor. Proceedings of the Annual Reliability and Maintainability Symposium, Lake Buena Vista, 24-27 January 2011, 1-5.

[17] Wohlgemuth, J.H. (1993) Testing for Module Warranties. NREL/DOE Photovoltaic Performance and Reliability Workshop, NREL/CP-410-6033 DE94000236, Golden, CO, 200-205. 
[18] Kern, G. (1999) SunSineTM300: Manufacture of an AC Photovoltaic Module. Final Report, Phases I \& II NREL/SR520-26085.

[19] Charki, A., Laronde, R. and Bigaud, D. (2013) Accelerated Degradation Testing of a Photovoltaic Module. Journal of Photonics for Energy, 3, Article ID: 033099. http://dx.doi.org/10.1117/1.JPE.3.033099

[20] Laronde, R., Charki, A. and Bigaud, D. (2012) Lifetime Estimation of a Photovoltaic Module Subjected to Corrosion Due to Damp Heat Testing. Journal of Solar Energy Engineering, 135, Article ID: 021010.

http://dx.doi.org/10.1115/1.4023101

[21] Laronde, R. (2009) Solutions Photovoltaiques dans le Ba^timent. GINGER CATED.

[22] Oreski, G. and Wallner, G.M. (2005) Aging Mechanisms of Polymeric Films for PV Encapsulation. Solar Energy, 79, 612-617. http://dx.doi.org/10.1016/j.solener.2005.02.008

[23] Reis, A.M., Coleman, N.T., Marshall, M.W., Lehman, P.A. and Chamberlain, C.E. (2002) Comparison of PV Module Performance before and after 11-Years of Field Exposure. Proceedings of the 29th IEEE Photovoltaic Specialists Conference, New Orleans, 20-24 May 2002, 1432-1435. http://dx.doi.org/10.1109/pvsc.2002.1190878

[24] Sakamoto, S. and Oshiro, T. (2003) Field Test Results on the Stability of Crystalline Silicon Photovoltaic Modules Manufactured in the 1990's. Proceedings of the 3rd World Conference on Photovoltaic Energy Conversion, Osaka, 11-18 May 2003, 1888-1891.

[25] Osterwald, C.R., Benner, J.P., Pruett, J., Anderberg, A., Rummeland, S. and Ottoson, L. (2003) Degradation in Weathered Crystalline-Silicon PV Modules Apparently Caused by UV Radiation. Proceedings of the 3rd World Conference on Photovoltaic Energy Conversion, Osaka, 11-18 May 2003, 2911-2915.

[26] Raghuraman, B., Laksman, V., Kuitche, J., Shisler, W., Tamizhani, G. and Kapoor, H. (2006) An Overview of SMUDs Outdoor Photovoltaic Test Program at Arizona State University. Proceedings of the 4th IEEE World Conference on Photovoltaic Energy Conversion, Waikoloa, 7-12 May 2006, 2214-2216.

[27] Marion, B. and Adelstein, J. (2003) Long-Term Performance of the SERF PV Systems. Proceedings of the NCPV and Solar Program Review Meeting, Denver, 24-26 March 2003, 1-3.

[28] Vazquez, M. and Ignacio, R.S. (2008) Photovoltaic Module Reliability Model Based on Field Degradation Studies. Progress in Photovoltaics: Research and Applications, 16, 419-433. http://dx.doi.org/10.1002/pip.825

[29] Glasstone, S., Laidler, K.J. and Eyring, H. (1941) Theory of Rate Processes. McGraw-Hill, New York.

[30] Eyring, H. (1980) Basic Chemical Kinetics. Wiley, New York.

[31] Weston, R. and Schwarz, H.A. (1972) Chemical Kinetics. Prentice Hall, Englewood Cliffs.

[32] Boccaletti, G., Borri, F.R., D’Esponosa, F. and Ghio, E. (1989) Chapter 11: Accelerated Tests. In: Pollino, E., Ed., Microelectronic Reliability —Volume 2: Integrity Assessment and Assurance, Artech House, Norwood.

[33] Klinger, D.J. (1991) On the Notion of Activation Energy in Reliability: Arrhenius, Eyring and Thermodynamics. Proceedings of the Annual Reliability and Maintainability Symposium, Orlando, 29-31 January 1991, 295-300.

[34] Nelson, W. (1990) Accelerated Testing: Statistical Models, Test Plans and Data Analyses. Wiley, New York. http://dx.doi.org/10.1002/9780470316795

[35] Mann, N.R., Schafer, R.E. and Singpurwalla, N.D. (1974) Methods for Statistical Analysis of Reliability and Life Data. Wiley, New York.

[36] Peck, D.S. (1986) Comprehensive Model for Humidity Testing Correlation. Proceedings of the IEEE International Reliability Physics Symposium, Anaheim, 1-3 April 1986, 44-50. http://dx.doi.org/10.1109/irps.1986.362110

[37] Peck, D.S. and Zierdt Jr., C.H. (1974) The Reliability of Semiconductor Devices in the Bell System. Proceedings of the IEEE, 62, 185-211. http://dx.doi.org/10.1109/proc.1974.9408

[38] Joyce, W.B., Liou, K.-Y., Nash, F.R., Bossard, P.R. and Hartman, R.L. (1985) Methodology of Accelerated Aging. AT\&T Technical Journal, 64, 717-764. http://dx.doi.org/10.1002/j.1538-7305.1985.tb00446.x

[39] Gillen, K.T. and Mead, K.E. (1980) Predicting Life Expectancy and Simulating Age of Complex Equipment Using Accelerated Aging Techniques. National Technical Information Service, US Department of Commerce, Springfield.

[40] LuValle, M.J., Welsher, T.L. and Mitchell, J.P. (1986) A New Approach to the Extrapolation of Accelerated Life Test Data. Proceedings of the Fifth International Conference on Reliability and Maintainability, Biarritz, 6-10 October 1986, 620-635.

[41] James, T.H., Ed. (1977) The Theory of the Photographic Process. 4th Edition, Macmillan, New York.

[42] Blum, H.F. (1959) Carcinogenesis by Ultraviolet Light. Princeton University Press, Princeton.

[43] Kojima, T. and Yanagisawa, T. (2004) The Evaluation of Accelerated Test for Degradation a Stacked a-Si Solar Cell and EVA Films. Solar Energy Materials \& Solar Cells, 81, 119-123. http://dx.doi.org/10.1016/j.solmat.2003.09.003 
[44] Oreski, G. and Wallner, G.M. (2009) Evaluation of the Aging Behavior of Ethylene Copolymer Films for Solar Applications under Accelerated Weathering Conditions. Solar Energy, 83, 1040-1047. http://dx.doi.org/10.1016/j.solener.2009.01.009

[45] Wohlgemuth, J.H. and Kurtz, S. (2011) Reliability Testing beyond Qualification as a Key Component in Photovoltaic’s Progress toward Grid Parity. Proceedings of the IEEE International Reliability Physics Symposium Monterey, Monterey, 10-14 April 2011, 5E.3.1-5E.3.6. http://dx.doi.org/10.1109/irps.2011.5784534

[46] Zimmerman, C.G. (2008) Time Dependent Degradation of Photovoltaic Modules by Ultraviolet Light. Applied Physics Letter, 92, Article ID: 241110. http://dx.doi.org/10.1063/1.2947589

[47] Martin, J.W., Saunders, S.C., Floyd, F.L. and Wineburg, J.P. (1996) Methodologies for Predicting the Service Lives of Coating Systems. Federation of Societies for Coatings Technology, Blue Bell.

[48] Meeker, W.Q. and Escobar, L.A. (1998) Statistical Methods for Reliability Data. Wiley, New York.

[49] Boyko, K.C. and Gerlach, D.L. (1989) Time Dependent Dielectric Breakdown at $210 \AA \AA$ Oxides. Proceedings of the 27th Annual International Reliability Physics Symposium, Phoenix, 11-13 April 1989, 1-8. http://dx.doi.org/10.1109/relphy.1989.36309

[50] Escobar, L.A. and Meeker, W.Q. (2006) A Review of Accelerated Test Models. Statistical Science, 21, 552-577. http://dx.doi.org/10.1214/088342306000000321

[51] Kurtz, S., Whitfield, K., Tamizhmani, G., Koehl, M., Miller, D., Joyce, J., Wohlgemuth, J., Bosco, N., Kempe, M. and Zgonena, T. (2011) Evaluation of High-Temperature Exposure of Photovoltaic Modules. Progress in Photovoltaics: Research and Applications, 19, 954-965. http://dx.doi.org/10.1002/pip.1103

[52] Burch, D., Martin, J.W. and Van Landingham, M.R. (2002) Computer Analysis of a Polymer Coating Exposed to Field Weather Conditions. Journal of Coatings Technology, 74, 75-86. http://dx.doi.org/10.1007/bf02720143 